\title{
HERRAMIENTAS PARA SIMULACIÓN DE MATRICES PRODUCTIVAS ÓPTIMAS DEL ECUADOR
}

\author{
ISSN 2219-6722 \\ ISSNE 2222-2707 \\ FLORES-TAPIA CARLOS ERNESTO Pontificia Universidad \\ Católica del Ecuador, Sede Ambato- Ecuador \\ cflores@pucesa.edu.ec \\ FLORES-CEVALLOS KARLA LISSETTE Fundación \\ Los Andes - Ecuador \\ florestapiacarlos@yahoo.com
}

\section{RESUMEN}

El gobierno ecuatoriano presidido por el Eco. Rafael Correa impulsa el cambio de la matriz productiva como una política de Estado para superar el modelo económico primario. Prioriza 14 sectores y 5 industrias estratégicos para modelar el cambio. La matriz propuesta por el gobierno no se sustenta en métodos cuantitativos que permitan calcular e interpretar las interdependencias de los diversos sectores de la economía; sino en apreciaciones subjetivas relacionadas con su posible potencial de mercado. El estudio contempla el análisis de la percepción de los sectores productivos de la provincia de Tungurahua con respecto a la priorización propuesta por el gobierno y el desarrollo de herramientas informáticas integradas en el "Simulador de la Matriz Insumo Producto" que permite calcular el impacto económico de cada rama del Sistema de Clasificación Industrial de América del Norte 2013 (SCIAN) en la economía total, en la propia rama y en otras ramas afectadas; y evaluar las distintas alternativas de matrices óptimas aplicando la Matriz de Leontief. Se recopila información existente en bases de datos gubernamentales y se establecen los criterios de análisis que permiten procesar la información con algoritmos matemáticos que vinculan herramientas informáticas. Finalmente se está en condiciones de proyectar el impacto de los resultados a nivel de producción, remuneraciones y puestos de trabajo, inducidos por cambios en alguno de los componentes de la demanda final. El estudio resulta prioritario porque aporta una visión estratégica para la toma de decisiones de la inversión pública y privada en el Ecuador sustentada en herramientas técnicas cuantitativas.

Palabras clave: Economía aplicada, Desarrollo Territorial, Matriz Insumo Producto, Herramientas de simulación, Planificación, Productividad, Competitividad, Gestión Estratégica, Métodos cuantitativos. 


\title{
SIMULATION TOOLS FOR OPTIMAL PRODUCTIVE DIES OF ECUADOR
}

ISSN 2219-6722

ISSNE 2222-2707

\author{
FLORES-TAPIA CARLOS ERNESTO Pontificia Universidad \\ Católica del Ecuador, Sede Ambato- Ecuador \\ cflores@pucesa.edu.ec \\ FLORES-CEVALLOS KARLA LISSETTE Fundación \\ Los Andes - Ecuador \\ florestapiacarlos@yahoo.com
}

\begin{abstract}
The Ecuadorian government headed by Eco. Rafael Correa promotes changing the production model as a state policy to overcome the primary economic model. 14 priority sectors and five strategic industries to model change. The matrix proposed by the government is not based on quantitative methods to calculate and interpret the interdependencies of the various sectors of the economy; but on subjective judgments related to possible market potential. The study includes an analysis of the perception of the productive sectors of the province of Tungurahua regarding the prioritization proposed by the government and the development of integrated in the "Simulator input-output matrix" used to calculate the economic impact of IT tools each branch of the industry Classification System North 2013 (NAICS) America in the overall economy, the industry itself in other branches affected; and assess the various options for optimal matrices applying the Leontief. (Nobel Prize 2016). Existing information is collected on government databases and the analysis criteria that allow process information with mathematical algorithms linking tools are established. Finally he is able to project the impact of the results in terms of production, wages and jobs, induced by changes in any of the components of final demand. The study is a priority because it provides a strategic vision for decision-making in public and private investment in Ecuador quantitative techniques supported by tools.
\end{abstract}

Keywords: Applied Economics, Territorial Development, Input-Output Matrix, Simulation Tools, Planning, Productivity, Competitiveness, Strategic Management, Quantitative Methods. 


\section{INTRODUCCIÓN}

El gobierno nacional identifica catorce sectores y cinco industrias estratégicos para el cambio de matriz productiva del Ecuador (Secretaría Nacional de Planificación y Desarrollo 2012), lo hace sin un sustento técnico matemático que permita medir el impacto en el crecimiento económico que puede reflejarse en los niveles de variación, por ejemplo, en la producción, remuneraciones y empleo. El gobierno prioriza este conjunto de sectores e industrias, en primer lugar, con criterios subjetivos tales como su posible potencial de mercado y su articulación a la política de sustitución de importaciones. Adicionalmente, a priori, el gobierno en funciones establece un modelo económico endógeno como el óptimo para el Ecuador. Y, en tercer término, sigue primando en el Ecuador la cultura del inmediatismo en el desarrollo económico versus la planificación prospectiva.

Este planteamiento puede traer como consecuencias el debilitamiento de sectores productivos que tienen resultados y proyecciones de crecimiento y/o el impulso de sectores productivos en decadencia, generando ineficiencias económicas.

En el plano social, algunos colectivos sociales no están plenamente de acuerdo con la priorización de sectores e industrias realizada por el gobierno nacional, resquebrajándose la gobernanza del país y la profundización democrática.

Este estudio aporta un instrumento para evaluar y proyectar escenarios económicos de las distintas alternativas de priorización de los sectores productivos en función de la matriz óptima con el fin de tomar la mejor decisión para el país, en beneficio de la ciudadanía y de los inversionistas públicos y privados. Incluye técnicas de simulación con métodos cuantitativos con el fin de comparar y obtener las alternativas de matriz productiva que mejoran la propuesta por el gobierno.

El estudio intenta responder a la pregunta: ¿cómo calcular alternativas de matriz productiva óptima del Ecuador utilizando métodos cuantitativos? Asimismo, pretende probar que la percepción de los colectivos sociales concuerda parcialmente con la propuesta gubernamental de priorización de sectores e industrias estratégicos para el cambio de la matriz productiva del Ecuador.

El objetivo general del estudio es desarrollar herramientas de simulación para la determinación de matrices productivas óptimas del Ecuador. Los objetivos específicos son:

- Fundamentar teóricamente el cálculo de la matriz óptima y las proyecciones de los impactos económicos y sociales en el Ecuador.

- Analizar la percepción de los sectores productivos y de la ciudadanía con respecto a la priorización de sectores e industrias estratégicas para el cambio de la matriz productiva propuesta por el gobierno nacional.

- Operativizar un sistema informático que calcule los efectos en las matrices de insumos intermedios y en la demanda final ocasionadas por variaciones en los niveles de producción, utilizando la Matriz de Leontief (Nobel Prize 2016).

A continuación, se revisa la literatura previa y se redacta el estado del arte. Después se detalla la metodología. En la siguiente parte se exponen los resultados y la discusión de los mismos. Finalmente se presentan las conclusiones e implicaciones, así como las áreas de investigación futura.

1 Una característica de la investigación de operaciones es que intenta encontrar una mejor solución —llamada solución óptima— Se dice una mejor solución y no la mejor solución porque es posible que existan muchas soluciones que puedan considerarse como las mejores (Hillier \& Lieberman 2002). 
Herramientas para Simulación de Matrices

ProduCtivas OPTIMAS DEL ECUADOR

\section{MARCO TEÓRICO}

El método de análisis input-output, también conocido como Matriz Insumo Producto, (Tarancón Morán 2011) desarrollado por el profesor Wassily Leontief es un instrumento que permite medir las interdependencias de los diversos sectores económicos. Se fundamenta en la premisa de que el sistema económico responde a una complejidad de industrias mutuamente interrelacionadas, de tal manera que, toda industria provee su producción y recibe insumos o materia prima, a su vez, para su producción.

El cimiento de la elaboración de las primeras tablas input-output por parte de Leontief (Cañada \& Toledo 2001) está comprendido entre los años 1932 y 1939; se aplican a la economía americana correspondiente a los años 1919 y 1929. Los avances de sus trabajos son publicados en los años 1936 y 1937. En 1941 culmina su investigación con la publicación de su obra más conocida, The Structure of American Industry.

A la par que se desarrollan los primeros análisis input-output se realizan estudios en temas tales como las curvas de indiferencia y el comercio internacional, así como también sobre las corrientes de la teoría económica, enfatizando los enfoques de Keynes, de la escuela de Cambridge y del marxismo. De 1944 a 1946 aparecen una serie de publicaciones referidas a investigaciones del denominado modelo abierto input-output, en las cuales se identifican algunos campos de aplicación de este modelo a futuro.

En la década de los cincuenta (Cañada \& Toledo 2001) se tienen importantes aportes al análisis input-output, como por ejemplo la segunda edición de la Estructura de economía americana (1951), así como artículos en obras colaborativas y recopilación de trabajos de economía americana vinculados con Harvard Project.

Las evaluaciones del impacto económico de los programas de desarme analizados con los modelos input-output en Estados Unidos y en el ámbito internacional, en los años sesenta, es financiada por la Organización de las Naciones Unidas. La década de los setenta marca el inicio del ciclo de conferencias internacionales sobre el método de análisis input-output, o también denominado Matriz Insumo Producto (Márquez González 2014), con el fin de intercambiar experiencias y difundir el avance teórico y aplicaciones.

La investigación científica (Fontela \& Pulido 2005), presenta la evolución cronológica de las investigaciones desde el año 1960 al 2004; se destacan aspectos teóricos, metodológicos, empíricos y aplicaciones y se hace referencia a la creación de la Asociación Internacional Input-output en el año 1986.

Este antecedente histórico motiva a los Bancos Centrales de algunos países a elaborar fichas técnicas de estimación utilizando la Matriz Insumo Producto. La Matriz de Leontief se presenta como herramienta idónea para el análisis macroeconómico, además su utilidad radica en la implementación de las mejoras en el marco contable del Sistema de Cuentas Nacionales. Asimismo, permite profundizar en la descripción y evaluación de las relaciones interdependientes entre los diferentes sectores productivos de la economía, principalmente todas aquellas transacciones intermedias reales entre los mismos. Sus datos usualmente son fáciles de ensamblar en los modelos macroeconómicos destinados a analizar las relaciones entre demanda final y los niveles de producción por actividades económicas. Es el caso por ejemplo del Banco Central de Chile, (Banco Central de Chile 2001), Banco Central de la República Argentina, (Instituto Nacional de Estadística y Censos Argentina. Ministerio de Economía. Secretaría de Política Económica 2001), Banco Central de la República Bolivariana de Venezuela, (Banco Central de Venezuela 2012), Estados Unidos de Norteamérica, (Aroche et al. 2012), Banco Central de la República de Colombia, (Departamento Nacional de Estadística de Colombia 2014), entre otros. El alcance que tienen dichos estudios es eminentemente descriptivo.

Otra de las definiciones (Schuschny 2005) de las tablas de insumo-producto es que son un conjunto integrado de matrices, que muestran el equilibrio entre la oferta y utilización de bienes y servicios (productos). Estas matrices proporcionan un análisis detallado del proceso de producción y la utilización de los bienes y servi- 
cios que se producen en un país (o región) o que se importan del resto del mundo, y del ingreso generado en dicha producción por las diversas actividades económicas. Para su construcción se requiere poner en marcha un conjunto de actividades como la de centralizar, analizar y procesar información básica de múltiples fuentes como pueden ser: censos económicos, agropecuarios, censos de población y vivienda, encuestas de gastos e ingresos de los hogares, registros administrativos y, fundamentalmente, los Sistemas de Cuentas Nacionales. En el escenario nacional, el Banco Central del Ecuador publica en mayo del 2014 los resultados del cálculo de la Matriz Insumo Producto (Banco Central del Ecuador 2014) correspondiente al año 2010, se muestran en las matrices simétrica e inversa los valores de los cálculos de las interrelaciones sectoriales utilizando la Matriz de Leontief. Los resultados describen sintéticamente la situación de la economía nacional y cuantifican los efectos directos e indirectos que las industrias tienen sobre la economía del país con el fin de facilitar y ser una herramienta en la toma de decisiones de la política pública.

La matriz productiva (Secretaría Nacional de Planificación y Desarrollo 2012) promocionada por el gobierno liderado por el Eco. Rafael Correa tiene como rasgo distintivo básico su estructuración en torno a una función de producción donde la tasa de crecimiento depende básicamente del stock de tres factores: capital físico, capital humano y conocimientos (o progreso técnico), que pueden ser objeto de acumulación y, además, generan externalidades. Descartando con ello alternativas sinérgicas que pueden derivar en mejores resultados a mediano y largo plazo para la economía ecuatoriana.

Desde la perspectiva optimista se puede decir que las unidades productivas nacionales pueden aprovechar oportunidades derivadas de la priorización que hace el gobierno central de sectores estratégicos (Jaramillo 2014), más aún cuando se lo hace en el marco de una política de sustitución de importaciones. La clave de este proceso, para las unidades productivas, reside en focalizarse en la diversificación de la producción, generación de valor agregado y el incremento de la oferta exportable. Las empresas tendrán que estar ligadas a los sectores estratégicos para encontrar oportunidades y, con ello, definir las inversiones necesarias para apalancar un modelo productivo empresarial que apuntale la estructura económica nacional, incremente el empleo y permita una mejor distribución de la riqueza.

Cabe indicar que esta investigación está clasificada en la Nomenclatura Internacional de la Organización de las Naciones Unidas para la Educación, la Ciencia y la Cultura -UNESCO (Ministerio de Economía y Competitividad del Gobierno de España 2016) para el campo de la Ciencias Económicas, en la disciplina de Econometría, y subdisciplina Modelos Econométricos (530202) y en la disciplina Contabilidad Económica, y su disciplina Input-Output (530304).

\section{METODOLOGÍA}

La investigación se respalda en la metodología Sistema Europeo de Cuentas (SEC), la cual aborda el análisis de los sistemas económicos desde dos perspectivas, (Carrasco Canals 1999).

- Análisis Funcional: estudio de la actividad de producción y del equilibrio entre oferta (recursos) y demanda (empleos) de los productos.

- Análisis Estructural: estudio de los modelos económicos que favorezcan la implementación de alternativas óptimas de matriz productiva.

El estudio contempla también técnicas cuantitativas (Hillier \& Lieberman 2002; Taha 2012; Winston 2004) que se ajustan a la Metodología de la Investigación de Operaciones; en la cual se consideran las siguientes etapas:

- Definición del problema de interés y recolección de datos relevantes.

- Formulación de un modelo matemático que represente el problema. 
- Desarrollo de un procedimiento basado en computadora para derivar una solución para el problema a partir del modelo.

- Prueba del modelo y mejoramiento de acuerdo con las necesidades.

- Preparación para la aplicación del modelo prescrito por la administración.

- Implementación.

En la fundamentación teórica para el cálculo de la matriz óptima y las proyecciones de los impactos económicos y sociales, consignada en el apartado del Marco teórico, se aplican técnicas de búsqueda y gestión de la información científica sustentada en bases de datos y recursos bibliográficos en formatos impresos y digitales.

En el análisis de percepción de los sectores productivos y de la ciudadanía con respecto a la priorización de sectores e industrias estratégicos para el cambio de la matriz productiva propuesta por el gobierno nacional se aplica una encuesta a la ciudadanía ${ }^{2}$ con una muestra de 384 personas de una población de 504.583 habitantes y otra encuesta a 130 empresarios de la provincia de Tungurahua. En la recolección, organización de datos y en la presentación, análisis e interpretación de resultados se emplean técnicas estadísticas.

Para desarrollar el sistema informático denominado "Simulador de la Matriz Insumo Producto" que calcula los efectos en las matrices de insumos intermedios y en la demanda final ocasionadas por variaciones en los niveles de producción, utilizando la Matriz de Leontief y la metodología RAS ${ }^{3}$, se consideran los siguientes pasos:

- Levantamiento de la información para la estructuración de la base de datos.

- Creación de la base datos.

- Definición del algoritmo de cálculo.

- Desarrollo de los formularios para los distintos ítems del sistema.

- Auditoría de que la información generada.

- Implementación del sistema denominado Simulador: Matriz Insumo Producto.

De acuerdo a Schuschny A. (Schuschny 2005), el cálculo de la Matriz Insumo Producto con el método de la Matriz de Leontief se basa en los supuestos de homogeneidad sectorial, invariancia de los precios relativos, hipótesis de la proporcionalidad, hipótesis de aditivita, relación de precios presente en el año en que se elabora la matriz.

\section{RESULTADOS Y DISCUSIÓN}

\subsection{Percepción de la ciudadanía y de los sectores productivos de la provincia de Tungurahua}

Los sectores productivos consideran que de los 15 sectores estratégicos priorizados por el gobierno nacional para el cambio de la matriz productiva se tiene que apoyar particularmente: alimentos frescos y procesados, energías renovables, turismo, tecnología, biotecnología (biomedicina), industria farmacéutica, vehículos-automotores-carrocerías y partes, confecciones y calzado, servicios ambientales; adicionalmente es necesario impulsar otros sectores no priorizados como son: agricultura, ganadería, comercio, emprendimientos, artesanía, minería, floricultura. En cuanto a las 5 industrias priorizadas por el gobierno nacional para el cambio de la matriz productiva, los sectores productivos jerarquizan la importancia en este orden: refinería, petroquímica, siderurgia, metalurgia, astillero.

2 Para el cálculo del tamaño de la muestra se considera el 5\% de error. Nivel de Confianza 95\%. Varianza 0.25. Valor tipificado "z" de 1,96, doble cola.

3 El método biproporcional sintético de ajuste denominado RAS, en el marco del análisis insumo-producto, parte de una matriz de transacciones interindustriales $Z 0$ y de un vector de producción efectiva w0 que conjuntamente permiten definir la matriz A0 de coeficientes técnicos (Parra \& Pino 2003).

106 Revista Economía y Administración (E\&A) / Vol. 6 
Figura No. 1. Otros sectores productivos que deben ser considerados en el cambio de matriz productiva

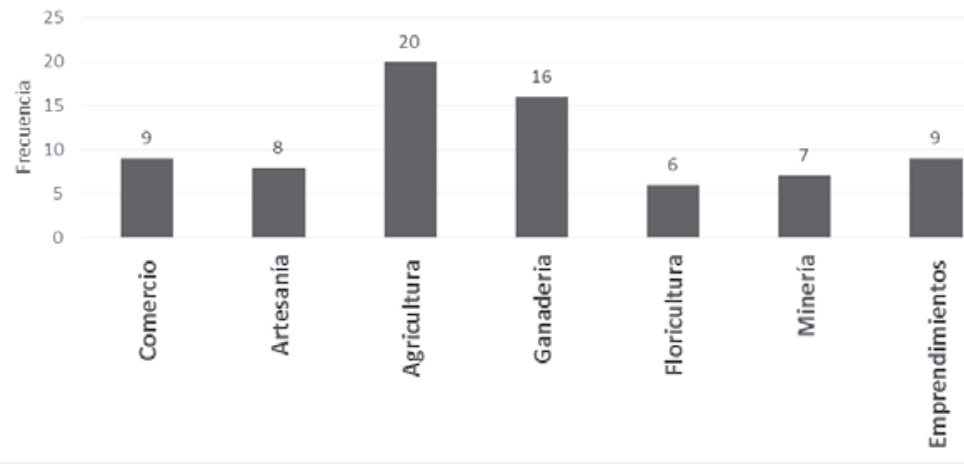

Elaborado por: Los autores.

Fuente: Encuesta a empresarios

La ciudadanía coincide con la priorización del gobierno nacional de los sectores alimentos frescos y procesados, turismo, confecciones y calzado, vehículos-automotores-carrocerías y partes; a la vez que consideran que es necesario impulsar adicionalmente los sectores: agricultura, comercio, ganadería, artesanía, transporte, construcción, minería, floricultura.

Figura No. 2. Otros sectores productivos que deben ser considerados en el cambio de matriz productiva

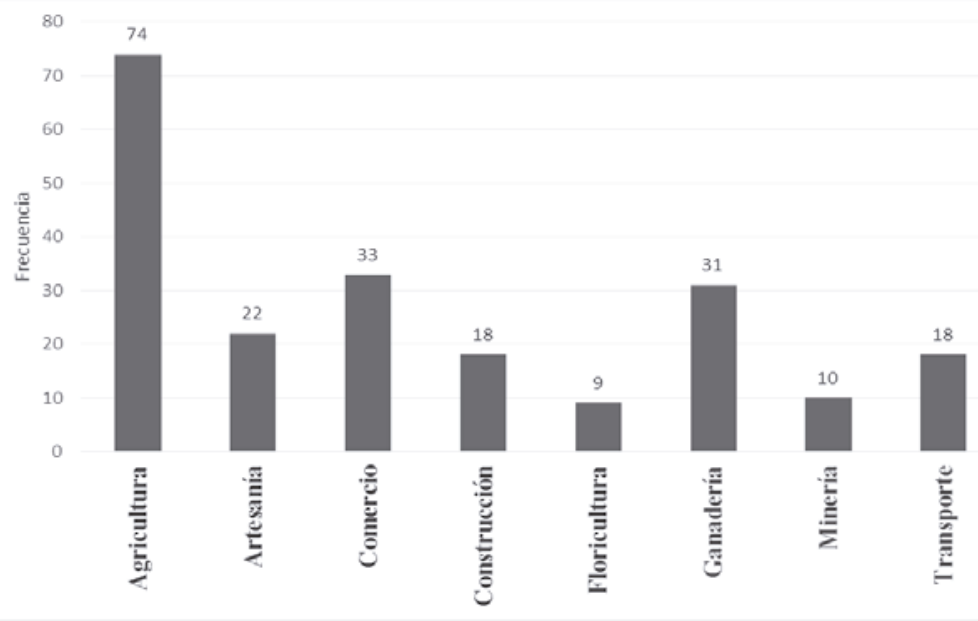

Elaborado por: Los autores.

Fuente: Encuesta a la ciudadanía

La percepción ciudadana y la de los empresarios coinciden en que el efecto de las políticas consideradas por el gobierno nacional para el cambio de la matriz productiva es de nivel medio; esto es, las políticas implementadas han sido medianamente efectivas.

Tanto para la ciudadanía como para los sectores productivos, las políticas públicas en las cuales el gobierno nacional tiene que enfatizar son: capacitación, financiamiento, reducción de cargas impositivas, innovación, inversiones, equidad social. Los resultados muestran también que es aún insuficiente el apoyo gubernamental para el cambio de la matriz productiva.

Tanto la ciudadanía como los sectores empresariales consideran necesario contar con una herramienta 
técnica de simulación para la toma de decisiones de inversión pública y privada en los distintos sectores productivos.

\subsection{Sistema informático "Simulador Matriz Insumo Producto"}

El resultado del desarrollo de un sistema denominado "Simulador Insumo-Producto tiene la siguiente estructura:

- Ítem matriz de insumos intermedios a precios básicos

Matriz de insumos intermedios original

Recalculo de matriz de insumos intermedios

- Ítem simulaciones

Por rama SCIAN

Impacto en la producción bruta total

Impacto en la rama modificada

Impacto en principales ramas afectadas

- Ítem proyecciones

Opción matriz de insumos intermedios proyectada

Las herramientas de desarrollo del sistema informático denominado "Simulador Matriz Insumo Producto" son las siguientes:

- Para el desarrollo del Front-end, se utiliza Visual Studio 2010, con el lenguaje de programación C\# y Aspnet 4.0

- Los componentes visuales del sistema son los controles Obout en la Enterprise Edition.

- El motor de base de datos es SQL Server 2008, versión express gratuita.

- La publicación de la plataforma en la web se realiza a través de Internet Information Server 7.0.

- La plataforma se ejecuta correctamente en los navegadores Google Chrome, Mozilla Firefox en todas las versiones, Safari e Internet Explorer a partir de la versión 8.0

Los datos utilizados en el modelamiento son tomados del informe de presentación de la Matriz Insumo Producto (MIP) del Banco Central del Ecuador, en la cual se identifican 71 sectores de la Economía ecuatoriana (Banco Central del Ecuador 2014). La MIP constituye una herramienta clave de la Contabilidad Nacional, se estructura sobre la base de dos tipos de tablas, de oferta utilización y la tabla simétrica del año 2012. La primera se refiere a la oferta total de los bienes y servicios de la economía y su utilización (demanda total). La segunda, la tabla simétrica, reordena y condensa la información de la tabla de Oferta Utilización, permitiendo el análisis económico con el método de Leontief, relacionando productos con productos o ramas de actividad con ramas de actividad. En este caso una matriz de 71 x 71 . 
Imagen No. 1. Aspecto de la página principal del Simulador Matriz Insumo Producto ${ }^{4}$

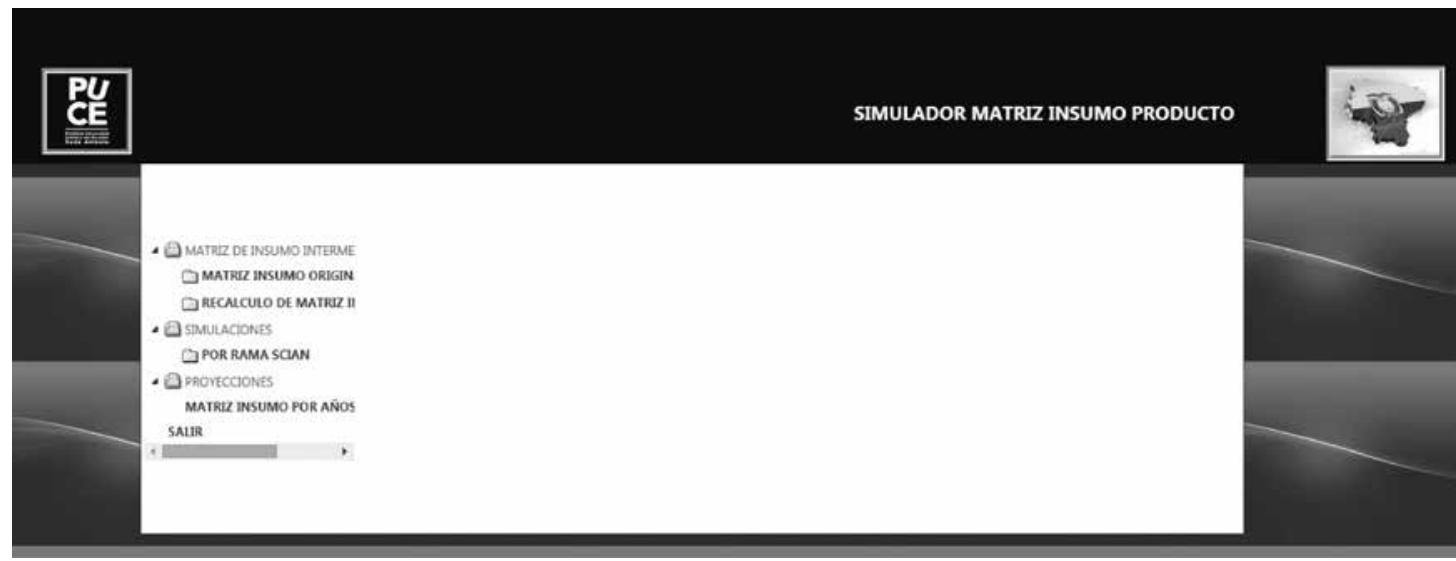

Elaborado por: Los autores.

Por ejemplo, el ítem simulaciones, por rama SCIAN, tiene el siguiente aspecto:

Imagen No. 2. Aspecto de la página "Îtem Simulaciones"

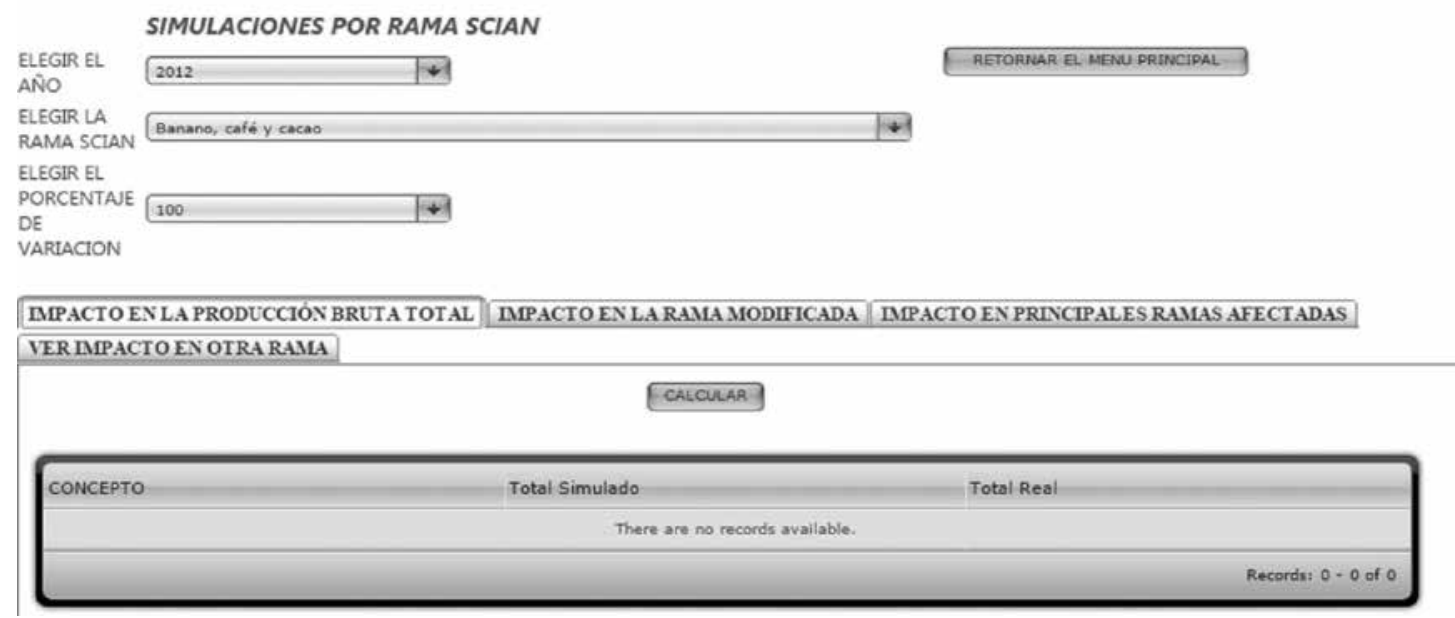

Elaborado por: Los autores.

Al elegir la rama SCIAN y seleccionar un porcentaje de variación, el usuario está en capacidad de visualizar los diversos modelos de simulación, como, por ejemplo, el impacto en la producción bruta total, el impacto en la rama modificada, el impacto en las principales ramas afectadas y, el impacto en otras ramas. 
Imagen No. 3. Aspecto de la página de salida del cálculo de "Impacto en la producción bruta total”

\begin{tabular}{|lll|}
\hline CONCEPTO & Total Simulado & Total Real \\
\hline PRODUCCION BRUTA & 29532004.15 & 29534341 \\
\hline TOTAL DE REMUNERACIONES & 46244077.693234 & 23238229.9966 \\
\hline TOTAL DE PUESTOS DE TRABAJO & 12914626.373632 & 6489761.9968 \\
\hline & & \\
\hline
\end{tabular}

Elaborado por: Los autores.

\section{CONCLUSIONES E IMPLICACIONES}

- La ciudadanía y los sectores productivos de la provincia de Tungurahua coinciden con el gobierno nacional en la priorización de algunos productos e industrias estratégicas; sin embargo, también consideran que se deben tomar en cuenta en el cambio de la matriz productiva nacional otros sectores.

- Se cuenta con una herramienta de simulación informatizada, que aplica la Matriz de Leontief y el método RAS, para el cálculo de los efectos en las matrices de insumos intermedios y en la demanda final ocasionados por variaciones en los niveles de producción, que permite tomar mejores decisiones en cuanto a la inversión pública y privada en función de los sectores productivos con mayor aporte al incremento de los indicadores macroeconómicos nacionales; consecuentemente se tiene la posibilidad de generar políticas públicas que fortalezcan y estimulen la inversión nacional y extranjera a favor de la productividad y competitividad en el mercado nacional e internacional.

- Se recomienda a los beneficiarios inmediatos: las Cámaras de Producción, de Comercio, de la Pequeña y Mediana Empresa, Secretaría Nacional de Planificación y Desarrollo, Ministerio de Industrias y Productividad del Ecuador, Inversionistas públicos y privados; y a los beneficiarios mediatos: Sectores productivos afines, Gobiernos Autónomos Descentralizados, Academia, y otras entidades del Estado Nacional; así como a la ciudadanía ecuatoriana comprometida en el cambio de matriz productiva el uso de esta herramienta de planificación que redundará en una mayor objetividad en la gestión de la política pública y en la gestión empresarial.

- El estudio abre la puerta para nuevas investigaciones que, con la aplicación de la Investigación de Operaciones, permitan generar un algoritmo que calcule la matriz óptima de factores de productividad y competitividad que maximice el crecimiento económico del Ecuador a corto, mediano y largo plazo. 


\section{REFERENCIAS BIBLIOGRÁFICAS}

Aroche, F., Frías, S. \& Torres, L., 2012. Matriz de insumo producto para América del Norte. Revista Realidad, Datos y Espacio. Revista Internacional de Estadística y Geografia, pp.70-89. Available at: http://www.inegi.org.mx/ prod_serv/contenidos/espanol/bvinegi/productos/integracion/especiales/revist-inter/revista_5/RDE_05_4a.html [Accessed February 17, 2016].

Banco Central de Chile, 2001. Matriz de Insumo Producto de la Economía Chilena 1996, Available at: http://si3.bcentral. cl/estadisticas/Principal1/Informes/CCNN/cdr/matriz1996.pdf [Accessed February 17, 2016].

Banco Central de Venezuela, 2012. Matriz Insumo Producto. BANCO CENTRAL DE VENEZUELA. Available at: http:// www.bcv.org.ve/cuadros/series/mip97/mip97.asp?id=425 [Accessed February 17, 2016].

Banco Central del Ecuador, 2014. Matriz Insumo Producto: Simétrica e Inversa, Available at: http://contenido.bce.fin.ec/ documentos/PublicacionesNotas/Catalogo/CuentasNacionales/Anuales/Dolares/PR MatrizInsumoProducto.pdf [Accessed February 17, 2016].

Cañada, A. \& Toledo, I., 2001. Leontief y España : Una reflexión sobre las tablas input / output y su relevancia para la economía. Información Comercial Española, ICE: Revista de economía, pp.51-76. Available at: http://www. revistasice.info/cachepdf/ICE_789_51-75_9AC809BE0BC9F4C5EA93608332BE4CC4.pdf.

Carrasco Canals, F., 1999. Fundamentos del sistema europeo de cuentas nacionales y regionales (SEC 1995), Madrid U6 - ctx_ver=Z39.88-2004\&ctx_enc=info\%3Aofi\%2Fenc\%3AUTF-8\&rfr_id=info:sid/ summon.serialssolutions.com\&rft_val_fmt=info:ofi/fmt:kev:mtx:book\&rft.genre=book\&rft. title $=$ Fundamentos + del + sistema + europeo + de + cuentas + nacionales $+y+$ regionales $+\% 28 \mathrm{SEC}+1995 \% 29 \& \mathrm{r}$ : Pirámide. Available at: http://uca.summon.serialssolutions.com/2.0.0/link/0/eLvHCXMwdV1LSwMxEB5Ke_ GmWPFRIUd76LJNNpvsuXYpFKFLey9JdgKC2EP w4L93JrZYRI-ZQ16EeXyZbwZAyaKc dIJQU1PpqX2VvnGaR2 NRnSIK4PtK51_ZtqN7jq1W-tuAKf-d3xFjlteFJh-UIu5NeS0k9ptVMVZXM-FZMsQ3Jll aC9hyGyBKxhguoaXlnkVjLcdkuj.

Departamento Nacional de Estadística de Colombia, 2014. Ficha Metodológica Cuentas Anuales de Bienes y Servicios -CABYS--, Available at: http://www.dane.gov.co/files/investigaciones/fichas/pib/Ficha_met_ctas_anua_bie_ ser_03_14.pdf [Accessed February 17, 2016].

Fontela, E. \& Pulido, A., 2005. Tendencias de la investigación en el análisis input-output. RAE: Revista Asturiana de Economía, pp.9-29. Available at: file://C:/Users/Usuario/Downloads/Dialnet-TendenciasDeLaInvestigacionEnElA nalisisInputoutput-2304670.pdf [Accessed February 17, 2016].

Hillier, F.S. \& Lieberman, G.J., 2002. Investigación de operaciones . Available at: http://uca.summon.serialssolutions. com/2.0.0/link/0/eLvHCXMwY2AwNtIz0EUrE8yB2cg8LR1Y9yYnpZoBWYZplkAhQ4Mkk0RgFwm0 OdktwDQw0DjE2zSQiQF2_x0oiBJBV17opRbDRy2MQIe-mYI2kFsam4BWcbnogfpbluagKwtMLMwgx_1ZwP mg00SARiBVG26CDCygrQRCDEypxSIMcvAjLZIz88805imkpCrkF6QWgbjA8ka.

Instituto Nacional de Estadística y Censos Argentina. Ministerio de Economía. Secretaría de Política Económica, 2001. Matriz insumo producto Argentina 1997, Available at: http://portalcdi.mecon.gov.ar/cgi-bin/wxis.exe/iah/ scripts/?IsisScript=iah.xis\&nextAction=lnk\&base=BIBLIO\&lang=es\&exprSearch=107061\&indexSearch= ZZ\&format=detailed.pft [Accessed February 17, 2016].

Jaramillo, J.P., 2014. Perspectiva económica del Ecuador:La matriz productiva 2014. PERSPECTIVA, p.16. Available at: http://investiga.ide.edu.ec/images/pdfs/2014abril/Perspectiva Abril 2014.9-12.pdf [Accessed February 17, 2016].

Márquez González, W., 2014. La Matriz de Leontief, Available at: http:/www.ehu.eus/Jarriola/Docencia/EcoEsp/matrizde-leontief.pdf [Accessed February 17, 2016].

Ministerio de Economía y Competitividad del Gobierno de España, 2016. Nomenclatura Internacional de la UNESCO para los campos de Ciencia y Tecnología. Available at: http:/www.idi.mineco.gob.es/portal/ site/MICINN/cmenuitem.8ce192e94ba842bea3bc811001432ea0/?vgnextoid=363ac948 7fb02210Vgn VCM1000001d04140aRCRD\&vgnextchannel=28fb282978 ea0210VgnVCM1000001034e20aRCRD [Accessed February 17, 2016].

Nobel Prize, 2016. Wassily Leontief - Biographical. Nobel Prize.org. Available at: http://www.nobelprize.org/nobel_ prizes/economic-sciences/laureates/1973/leontief-bio.html [Accessed February 18, 2016].

Parra, J.C. \& Pino, O., 2003. Obtención de una matriz insumo-producto a 20 sectores y análisis de los encadenamientos 
productivos para la región del Bío-Bío, base 2003. Horizontes empresariales, p.17. Available at: http://www. ubiobio.cl/miweb/webfile/media/42/version 7-1/economia.pdf [Accessed February 18, 2016].

Schuschny, A.R., 2005. Tópicos sobre el Modelo de Insumo-Producto: teoría y aplicaciones, Santiago de Chile: CEPAL. Available at: http://repositorio.cepal.org/bitstream/handle/11362/4737/S0501011_es.pdf?sequence=1 [Accessed February 17, 2016].

Secretaría Nacional de Planificación y Desarrollo, 2012. Transformación de la Matriz Productiva, Available at: http:// www.planificacion.gob.ec/wp-content/uploads/downloads/2013/01/matriz_productiva_WEBtodo.pdf [Accessed February 18, 2016].

Taha, H.A., 2012. Investigación de operaciones, México: Pearson Educación. Available at: http:/uca.summon. serialssolutions.com/2.0.0/link/0/eLvHCXMwdV3NCsIwDA5DL94UFf_ZC2x07ZzbWR2CFwfeR9el4MWJPoH v50OZ-DNE9JgePtoS8iVpkwAo6QvvyyZoNJaIEJOyIIoOyMm1xB1xpARqsdCc4k138yxT--08c-A9_46vSPPICx 8vddZCRfx0x122ExXyL66VXzO0ZFTyUij-ikh5peBKy2efnVrm7iIE-UEjaRs.

Tarancón Morán, M.Á., 2011. Técnicas de análisis económico input-output, ECU. Available at: http://uca.summon. serialssolutions.com/2.0.0/link/0/eLvHCXMwlV3LSsNAFB2KLnTlW2sV8gOJyTwyycqFtQiCWKjrMrlzAxFJikn B33Hp2k_ojzm3aUMpKLgKYRKYJDO5587ccw5jggehv_VP0LGVFJsyQB1nPM4MUKQwsdAOwhtaKRg9q FYTB7VuMeGf2ygZkVGdmCQ3dgKhss6y6DlANzaFax1kz_kROpTaUij_mlDD0a7AZm.

Winston, W.L., 2004. Investigación de operaciones: aplicaciones y algoritmos, México:

Thomson. Available at: http:/uca.summon.serialssolutions.com/2.0.0/link/0/ eLvHCXMwdV3NasJAEB6CvfRWUamtlryAIfub7FkNBS8NeJfJZgQvTTFP4Pv5 UN2JP5Wix9nDMLss8_EN8 80AKJmks385ATHgnnIetTVUZ0JK0tpgtVXSO9-N7S6-TFmq9cqUEVz23_ETIa-8SKi9Vi0Y8YRlzZ5Tmru4Fg nzLZex2iHkY3Gaq NnM2x4vIGN4gV6LCXoQ0T. 